\title{
Implementation and Comparative Study of Image Fusion Algorithms
}

\author{
Shivsubramani Krishnamoorthy \\ Centre for Excellence in Computational \\ Engineering, \\ Amrita University, Coimbatore, India.
}

\author{
K P Soman \\ Centre for Excellence in Computational \\ Engineering, \\ Amrita University, Coimbatore, India.
}

\begin{abstract}
Image Fusion is a process of combining the relevant information from a set of images, into a single image, wherein the resultant fused image will be more informative and complete than any of the input images. This paper discusses the implementation of three categories of image fusion algorithms - the basic fusion algorithms, the pyramid based algorithms and the basic DWT algorithms, developed as an Image Fusion Toolkit - ImFus, using Visual C++ 6.0. The objective of the paper is to assess the wide range of algorithms together, which is not found in the literature. The fused images were assessed using Structural Similarity Image Metric (SSIM) [10], Laplacian Mean Squared Error along with seven other simple image quality metrics that helped us measure the various image features; which were also implemented as part of the toolkit. The readings produced by the image quality metrics, based on the image quality of the fused images, were used to assess the algorithms. We used Pareto Optimization method to figure out the algorithm that consistently had the image quality metrics produce the best readings. An assessment of the quality of the fused images was additionally performed with the help of ten respondents based on their visual perception, to verify the results produced by the metric based assessment. Coincidentally, both the assessment methods matched in their raking of the algorithms. The Pareto Optimization method picked DWT with Haar fusion method as the one with the best image quality metrics readings. The result here was substantiated by the visual perception based method where it was inferred that fused images produced by DWT with Haar fusion method was marked the best $63.33 \%$ of times which was far better than any other algorithm. Both the methods also matched in assessing Morphological Pyramid method as producing fused images of inferior quality.
\end{abstract}

\section{Keywords}

Image Fusion, Principal Component Analysis, Pyramid Methods, Discrete Wavelet Transform, Image Quality Metrics, Pareto Optimality.

\section{INTRODUCTION}

Any piece of information makes sense only when it is able to convey the content across. The clarity of information is important. Image Fusion is a mechanism to improve the quality of information from a set of images. By the process of image fusion the good information from each of the given images is fused together to form a resultant image whose quality is superior to any of the input images.
This is achieved by applying a sequence of operators on the images that would make the good information in each of the image prominent. The resultant image is formed by combining such magnified information from the input images into a single image.

\subsection{Evolution of fusion techniques}

The evolution of the research work into the field of image fusion [9] [17] [24] [25] [26] can be broadly put into the following three stages

- $\quad$ Simple Image Fusion

- Pyramid Decomposition based fusion

- Discrete Wavelet transform based fusion

The eleven algorithms implemented and discussed here are such that all the three of the above categories are covered for assessment.

The primitive fusion schemes [9] perform the fusion right on the source images. This would include operations like averaging, addition, subtraction/omission of the pixel intensities of the input images to be fused. These methods often have serious side effects such as reducing the contrast of the image as a whole. But these methods do prove good for certain particular cases wherein the input images have an overall high brightness and high contrast. The primitive fusion methods considered were

- Averaging Method

- Select Maximum

- Select Minimum

With the introduction of pyramid transform [2] [3] [4] [5] [6] [9] in mid-80's, some sophisticated approaches began to emerge. People found that it would be better to perform the fusion in the transform domain. Pyramid transform appears to be very useful for this purpose. The basic idea is to construct the pyramid transform of the fused image from the pyramid transforms of the source images, and then the fused image is obtained by taking inverse pyramid transform. Pyramid transform could provide information on the sharp contrast changes, to which the human visual system is peticularly sensitive to and It could also provide both spatial and frequency domain localization. The pyramid fusion methods considered here were

\footnotetext{
${ }^{1}$ Shivsubramani Krishnamoorthy is currently a $\mathrm{PhD}$ student at MIND Lab, University of Maryland, College Park, MD 20742, USA.
} 
- Laplacian Pyramid

- Ratio-of-low-pass Pyramid

- Gradient Pyramid

- FSD Pyramid

- Morphological Pyramid etc.

Wavelet transforms [2] [6] [7] [9] [12] [16] [24] can be taken as a special type of pyramid decompositions. It retains most of the advantages for image fusion but has much more complete theoretical support. The couple of wavelet transform methods considered here are

- Haar Wavelet transform method

- Daubechies $(2,2)$ wavelet transform method.

\subsection{Objective}

The main objective of this paper is to

- Provide a study of 11 pixel based image fusion techniques.

- Discuss some of the implementation issues for the same.

- $\quad$ Assess the algorithms with 9 image quality metrics.

- Also assess the algorithms in terms of visual perception, with the help of 10 respondents and compare it with the assessment made with the metrics.

- We did not find in the literature a paper that encompasses such a wide range of image fusion methods. This paper aims in presenting these methods in a simplified manner, concentrating more on the implementation aspects of the algorithms.

We employed Pareto Optimization method [32] to pick out the algorithm for which the quality metrics consistently produced the best readings. The methods projected DWT with Haar based fusion method as superior based on the image quality metric readings. The same was validated by the assessment performed based on visual perception which also saw DWT with Haar based fusion method being selected as the best $63.33 \%$ times; which was way more that the other fusion methods. Both the assessment technique also assessed Morphological Pyramid based Fusion method as the inferior methods of the eleven algorithms considered.

\section{IMAGE FUSION ALGORITHMS}

In this section we discuss the set of image fusion algorithms we considered, categorizing them under three subsections

\subsection{Simple Fusion Algorithms}

The trivial image fusion techniques mainly perform a very basic operation like pixel selection, addition, subtraction or averaging. These methods are not always effective but are at times critical based on the kind of image under consideration. The trivial image fusion techniques studied and developed as part of the project are

\subsubsection{Average Method}

Here, the resultant image is obtained by averaging every corresponding pixel in the input images.

\subsubsection{Select Maximum/Minimum Method}

A selection process if performed here wherein, for every corresponding pixels in the input images, the pixel with maximum/minimum intensity is selected, respectively, and is put in as the resultant pixel of the fused image.

\subsubsection{Principal Component Analysis Algorithm}

Principal component analysis (PCA) [29] [30] is a vector space transform often used to reduce multidimensional data sets to lower dimensions for analysis. It reveals the internal structure of data in an unbiased way. We provide below the stepwise description of how we used the PCA algorithm for fusion.

1. Generate the column vectors, respectively, from the input image matrices.

2. Calculate the covariance matrix of the two column vectors formed in 1

3. The diagonal elements of the $2 \times 2$ covariance vector would contain the variance of each column vector with itself, respectively.

4. Calculate the Eigen values and the Eigen vectors of the covariance matrix

5. Normalize the column vector corresponding to the larger Eigen value by dividing each element with mean of the Eigen vector.

6. The values of the normalized Eigen vector act as the weight values which are respectively multiplied with each pixel of the input images.

7. Sum of the two scaled matrices calculated in 6 will be the fused image matrix.

\subsection{Pyramid Fusion Algorithm}

The decade of 1980's saw the introduction of pyramid transform [2] [3] [4] [5] [6] [9] - a fusion method in the transform domain. An image pyramid [17] consists of a set of low pass or bandpass copies of an image, each copy representing pattern information of a different scale. At every level of fusion using pyramid transform, the pyramid would be half the size of the pyramid in the preceding level and the higher levels will concentrate upon the lower spatial frequencies. The basic idea is to construct the pyramid transform of the fused image from the pyramid transforms of the source images and then the fused image is obtained by taking inverse pyramid transform.

Typically, every pyramid transform consists of three major phases:

- Decomposition

- Formation of the initial image for recomposition.

- Recomposition

Decomposition is the process where a pyramid is generated successively at each level of the fusion. The depth of fusion or number of levels of fusion is pre decided. Decomposition phase basically consists of the following steps. These steps are performed 1 number of times, 1 being the number of levels to which the fusion will be performed.

- $\quad$ Low Pass filtering. The different pyramidal methods have a pre defined filter with which are the input images convolved/filtered with.

- Formation of the pyramid for the level from the filtered/convolved input images using Burt's method or Lis Method.

- The input images are decimated to half their size, which would act as the input image matrices for the next level of decomposition.

Merging the input images is performed after the decomposition process. This resultant image matrix would act as the initial input to the recomposition process. The finally 
decimated input pair of images is worked upon either by averaging the two decimated input images, selecting the first decimated input image or selecting the second decimated input image.

The recomposition is the process wherein, the resultant image is finally developed from the pyramids formed at each level of decomposition. The various steps involved in the recomposition phase are discussed below. These steps are performed 1 number of times as in the decomposition process.

- The input image to the level of recomposition is undecimated

- The undecimated matrix is convolved/filtered with the transpose of the filter vector used in the decomposition process

- The filtered matrix is then merged, by the process of pixel intensity value addition, with the pyramid formed at the respective level of decomposition.

- The newly formed image matrix would act as the input to the next level of recomposition.

- The merged image at the final level of recomposition will be the resultant fused image. The flow of the pyramid based image fusion can be explained by the following example.

Each of the pyramidal algorithms considered by us differ in the way the decomposition is performed. The Recomposition phase also differs accordingly. We discuss the pyramid algorithms we implemented below.

\subsubsection{Filter Subtract Decimate Pyramid}

As suggested by the very name of this algorithm, the decomposition phase consists of three steps:

- Low pass filtering using $\mathrm{W}=\left[\frac{1}{16}, \frac{4}{16}, \frac{6}{16}, \frac{4}{16}, \frac{1}{16}\right]$.

- Subtract the low pass filtered input images and form the pyramid

- Decimate the input image matrices by halving the number of rows and columns (we did by neglecting every alternate row and column).

The recomposition phase would include steps:

- Undecimating the image matrix by duplicating every row and column

- Low pass filtering with $2 * \mathrm{~W}$

- Matrix addition of the same with the pyramid formed in the corresponding level

\subsubsection{Laplacian Pyramid}

The Laplacian pyramidal method is identical to FSD pyramid except for an additional low pass filtering performed with $2 * \mathrm{~W}$. All the other steps are followed as in FSD pyramid.

\subsubsection{Ratio Pyramid}

The Ration pyramidal method is also identical to FSD pyramid except for, in the decomposition phase, after low pass filtering the input image matrices, the pixel wise ratio is calculated instead of subtraction as in FSD.

\subsubsection{Gradient Pyramid}

The decomposition process here would include the following steps:

- Two low pass filters are considered here $\mathrm{W}=$ $\left[\frac{1}{16}, \frac{4}{16}, \frac{6}{16}, \frac{4}{16}, \frac{1}{16}\right]$ and $\mathrm{V}=\left[\frac{1}{4}, \frac{2}{4}, \frac{1}{4}\right]$.
- Additional to this, four directional filters are applied on to the input image matrices.

$$
\begin{gathered}
\circ \text { Horizontal filter }\left[\begin{array}{ccc}
0 & 0 & 0 \\
1 & -2 & 1 \\
0 & 0 & 0
\end{array}\right] \\
\circ \text { Vertical filter }\left[\begin{array}{ccc}
0 & 1 & 0 \\
0 & -2 & 0 \\
0 & 1 & 0
\end{array}\right] \\
\left.\circ \begin{array}{ccc}
0 & 0 & 0.5 \\
0 & -1 & 0 \\
0.5 & 0 & 0
\end{array}\right] \\
\left.\circ \begin{array}{ccc}
0.5 & 0 & 0 \\
0 & -1 & 0 \\
0 & 0 & 0.5
\end{array}\right]
\end{gathered}
$$

- The rest of the steps are similar to that of FSD pyramid method.

\subsubsection{Morphological Pyramid}

The decomposition phase in this method consists of the following steps:

- Two levels of filtering are performed on the input image matrices - image opening and image closing. Image opening is a combination of image erosion followed by image dilation. Image closing is the other way round. A combination of image opening and image closing gets rid of noise in the image[31].

- $\quad$ The rest of the steps are as in FSD pyramid method

The recomposition phase would be similar to the FSD method except for the step where the low pass filter is applied on the image matrix. Instead, an image dilation is performed over the matrix.

\subsection{Discrete Wavelet Transform Method}

DWT[2][6][7][9][12][16][24] captures not only a notion of frequency content of the input, by examining it at different scales, but also temporal content; i.e. the times at which these frequencies occur. The DWT of a signal $\mathrm{x}$ is calculated by passing it through a series of filters . First the passing it through a series of filters. First the samples are passed through a low pass filter with impulse response $g$ resoluting in a convolution of the two:

$$
y[n]=(x * g)[n]=\sum_{k=-\infty}^{\infty} x[k] g[n-k]
$$

The signal is also decomposed simultaneously using a highpass filter $h$. The outputs give the detail coefficients (from the high-pass filter) and approximation coefficients (from the low-pass).

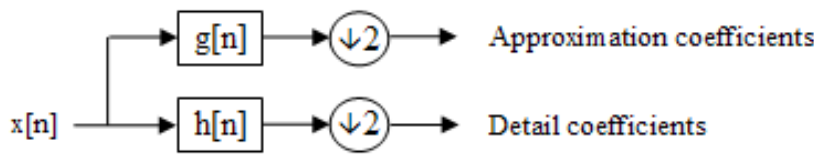

This decomposition is repeated to further increase the frequency resolution and the approximation coefficients decomposed with high and low pass filters and then down sampled. This is represented as a binary tree with nodes representing a sub-space with different time-frequency localization. The tree is known as a filter bank. 


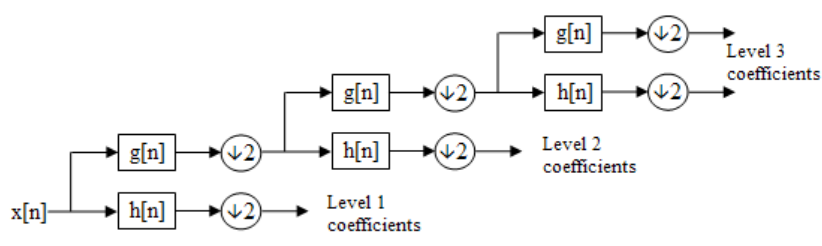

Since image is 2-D signal, we will mainly focus on the 2-D wavelet transforms. The following figures show the structures of 2-D DWT with 3 decomposition levels:

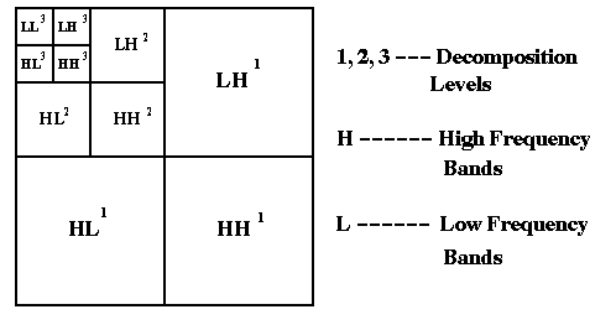

\subsubsection{DWT with Haar based fusion}

The Haar wavelet is the first known wavelet. The Haar wavelet $\psi(t)$ can be described as

and its scaling function $\varphi(t)$ can be described as

$$
\Psi(\mathrm{t})=\left\{\begin{array}{cl}
1 & 0 \leq t \leq \frac{1}{2} \\
-1 & \frac{1}{2} \leq t \leq 1 \\
0 & \text { Otherwise }
\end{array}\right.
$$

The $2 \times 2$ Haar matrix is associated with

$$
H_{2}=\left[\begin{array}{cc}
1 & 1 \\
1 & -1
\end{array}\right] .
$$

The filters, thus, considered here would be

$$
\begin{gathered}
\mathrm{F} 1=\left[\begin{array}{lllll}
* & 0.5 & * & 0.5 & *
\end{array}\right] \\
\mathrm{F} 2=\left[\begin{array}{lllll}
* & 0.5 & * & -0.5 & *
\end{array}\right]
\end{gathered}
$$

The couple of filters, when applied on the input images matrices, would produce 4 resultant matrices. The fourth matrix, which would consist of all the high frequencies, would act as the input matrix for the next level of decomposition. The other three matrices, consisting of the low frequencies, are used to produce 3 pyramids at each level. The pyramids are produced as in pyramidal method. The re-composition process is performed with the help of the three pyramids formed at each level of decomposition.

\subsubsection{DWT with DBSS $(2,2)$ based fusion}

The Daubechies wavelets are a family of orthogonal wavelets defining a discrete wavelet transform and characterized by a maximal number of vanishing moments for some given support. With each wavelet type of this class, there is a scaling function (also called father wavelet) which generates an orthogonal multiresolution analysis. In general the Daubechies wavelets are chosen to have the highest number A of vanishing moments, (this does not imply the best smoothness) for given support width $\mathrm{N}=2 \mathrm{~A}$, and among the
$2 \mathrm{~A}-1$ possible solutions the one is chosen whose scaling filter has extremal phase.

The filter considered here are :

$$
\begin{aligned}
& \mathrm{H} 1=\left[\begin{array}{lllll}
-1 & 2 & 6 & 2 & -1
\end{array}\right] \\
& \mathrm{H} 1=\left[\begin{array}{lllll}
-1 & -2 & 6 & -2 & -1
\end{array}\right] \\
& \mathrm{G} 1=\left[\begin{array}{lll}
2 & 4 & 2
\end{array}\right] \\
& \mathrm{G} 1=\left[\begin{array}{lll}
2 & 4 & 2
\end{array}\right]
\end{aligned}
$$

- The input image matrices of the level M1 and M2 are filtered into four bands each, say, A1, A2, A3, A4 and B1, B2, B3, B4 respectively.

- Calculate Z1 and Z2 by filtering M1 and M2 respectively with $\mathrm{G} 1$ filter at row level.

- A1 is calculated by filtering Z1 with transpose of G1 filter at column level.

- $\quad$ A2 is calculated by filtering Z1 with transpose of H1 filter at column level.

- Calculate Z1 and Z2 by filtering M1 and M2 again with $\mathrm{H} 1$ filter at row level.

- $\mathrm{A} 3$ is calculated by filtering $\mathrm{Z} 1$ with transpose of G1 filter at row level.

- $\quad$ A4 is calculated by filtering Z1 with transpose of H1 filter at row level.

- Similarly, B1, B2, B3 and B4 are calculated for M2 as in the case of M1.

- Three set of coefficient matrices (pyramids) are formed in this wavelet method, say, E1, E2 and E3.

The recomposition process was performed with superimposing the of the pyramids formed at each level to the undecimated image matrix at that level.

\section{IMAGE QUALITY METRICS}

Image Quality is a characteristic of an image that measures the perceived image degradation (typically, compared to an ideal or perfect image). Imaging systems like the fusion algorithm may introduce some amounts of distortion or artifacts in the signal, so the quality assessment is an important problem. Image Quality assessment methods can be broadly classified into two categories: Full Reference Methods (FR) and No Reference Method (NR). In FR, the the quality of an image is measure in comparison with a reference image which is assumed to be perfect in quality. NR methods do not employ a reference image.

The image quality metrics considered and implemented here fall in the FR category. In the following subsections, we discuss the SSIM and some other image quality metrics that we implemented in $\mathrm{VC}++$ to assess the quality of our fused images.

\subsection{Structural Similarity Index Metric}

The Structural Similarity Index measures [10] the similarity between two images. It is an improved version of traditional methods like PSNR and MSE. SSIM is considered to be one of the most effective and consistent metric.

SSIM is calculated based on two parameters - (i) K vector being a constant in the SSIM index formula with defualt value: $\mathrm{K}=\left[\begin{array}{ll}0.01 & 0.03\end{array}\right]$, (ii) $\mathrm{L}$ being the dynamic range of the images. In our case the default value of $L=255$. The values $\mathrm{C} 1$ and $\mathrm{C} 2$ are calculated based on the followinf formula:

$$
C_{1}=\left(K_{1} * L\right)^{2} \quad C_{2}=\left(K_{2} * L\right)^{2}
$$


$\mathrm{G}$ being a Guassian filter window with default value given in matlab as fspecial('gaussian', 11, 1.5), the input images A and $\mathrm{B}$ are low pass filtered with $\mathrm{G}$ giving $\mu_{1}$ and $\mu_{2}$ respectively. The filter operation or convolution operation performed is denoted by ".".

$$
\mu_{1}=A \cdot G \quad \mu_{2}=B \cdot G
$$

Then the values, $\sigma_{1}^{2}, \sigma_{2}^{2}$ and $\sigma_{12}^{2}$ are calculated based on the following formula.

$$
\begin{gathered}
\sigma_{1}^{2}=\left(A_{i j}^{2} \cdot G\right)-\mu_{1}^{2} \\
\sigma_{2}^{2}=\left(B_{i j}^{2} \cdot G\right)-\mu_{2}^{2} \\
\sigma_{12}^{2}=\left(A_{i j} B_{i j} \cdot G\right)-\mu_{1} \cdot * \mu_{2}
\end{gathered}
$$

Once the above values are calculated, finally the SSIM value is calculated based on the following formula:

$$
\operatorname{SSIM}=\text { mean }\left(\left(2 \times \mu_{1} \mu_{2}+C_{1}\right) \cdot *\left(2 \times \sigma_{12}+C_{2}\right) \cdot /\left(\left(\mu_{1}^{2}+\mu_{2}^{2}+C_{1}\right) \cdot *\left(\sigma_{1}^{2}+\sigma_{2}^{2}+C_{1}\right)\right)\right)
$$

The SSIM index is a decimal value between 0 and 1 . A value of 0 would mean zero correlation with the original image, and 1 means the exact same image. 0.95 SSIM, for example, would imply half as much variation from the original image as 0.90 SSIM. Through this index, image and video compression methods can be effectively compared.

\subsection{Laplacian Mean Squared Error}

$$
L M S E=\frac{\sum_{i=1}^{m} \sum_{j=1}^{n}\left(\nabla^{2} A-\nabla^{2} B\right)^{2}}{\sum_{i=1}^{m} \sum_{j=1}^{n}\left(\nabla^{2} A\right)^{2}}
$$

Laplacian Mean Square Error, as explained by the term, the the normal mean sqare error calculation. But the difference here is that the mean square error is calculated not based on the expected and obtained data but based on the laplacian value of the same. Thus, the laplacian of each of the values is calculated and then the net sum of the square of the error (difference) is calculated which is divided by the nte sum of the square of the laplacian of the expected data. Laplacian operator is defined by the following expression.

$$
\nabla^{2} u=\left(\frac{\partial^{2} u}{\partial x^{2}}+\frac{\partial^{2} u}{\partial y^{2}}\right)
$$

Let $u$ be defined asa function of $(x, y)$. Here $\mathrm{u}$ is the dependent variable $\mathrm{x}$ and $\mathrm{y}$ being independent variables. When there is a change in the variable $\mathrm{x}(\partial x)$, let the change in the dependent variable $u$ be $(\partial u)$. Then $\frac{\partial u}{\partial x}$ can be defined as the rate of change of the dependent variable along the $\mathrm{x}$-axis keeping the other dependent variable constant. Similarly $\frac{\partial u}{\partial y}$ is the rate of change in $u$ along the $y$-axis keeping the other dependent variable constant. $\frac{\partial^{2} u}{\partial x^{2}}$ is the rate of change in $\frac{\partial u}{\partial x}$ alond the x-axis, keeping y. $\frac{\partial^{2} u}{\partial y^{2}}$ can be defined similarly. In our

context, considering the images, $u$ being the pixel intensity value, is the function of the coordinate values $(x, y)$. Laplacian can be intepretted based on the context of consideration. In our case, the second rate of change of the intensity at perticular pixel in the images can be physically intepretted as the difference in the average of the neighboring pixel intensities and the intensity of the pixel under consideration. That is, each image pixel is subtracted from the average of the neighbouring pixels on the right, bottom, left and the top. This is considered the laplacian value of the perticular pixel. The del2, as the laplacian operator is denoted, is defined for a pixel $u(i, j)$ is defined as in the below expression.

$$
\nabla_{i j}^{2}=\frac{\left(u_{i, j+1}+u_{i, j-1}+u_{i+1, j}+u_{i-1, j}\right)}{4}-u_{i j}
$$

So, the Laplacian Mean Sqare Error is calculated as the ratio between the net sum of the square of the difference between the del 2 of the corresponding pixels of the perfect image and the fused image and the net sum of the square of the del 2 of the perfect image pixels. For an ideal situation, the fused and perfect image being identical, the LMSE value is supposed to be 0 . The error value which would exist otherwise would range from 0 to 1 .

\subsection{Other Simple Metrics}

Other simple image metrics were considered to measure the various features of the fused image to getter a better picture of the quality measurement. Assumptions made in the following equations are

$A$ - the perfect image

$B$ - the fused image to be assessed

$i$ - pixel row index

$j-$ pixel column index

\subsubsection{Mean Squared Error}

$$
M S E=\frac{1}{m n} \sum_{i=1}^{m} \sum_{j=1}^{n}\left(A_{i j}-B_{i j}\right)^{2}
$$

\subsubsection{Peak Signal to Noise Ratio}

$$
P S N R=10 \times \log _{10}\left(\frac{\text { peak }^{2}}{M S E}\right)
$$

Here, at pixel level, in a 8 bit greyscale image, the maximum possible value (peak) is having every bit as $1 \rightarrow 11111111$; which is equal to 255 .

\subsubsection{Average Difference}

$$
A D=\frac{1}{m n} \sum_{i=1}^{m} \sum_{j=1}^{n}\left(\left|A_{i j}-B_{i j}\right|\right)
$$




\subsubsection{Structural Content}

$$
S C=\frac{\sum_{i=1}^{m} \sum_{j=1}^{n}\left(A_{i j}\right)^{2}}{\sum_{i=1}^{m} \sum_{j=1}^{n}\left(B_{i j}\right)^{2}}
$$

\subsubsection{Normalized Cross Correlation}

$$
N C C=\frac{\sum_{i=1}^{m} \sum_{j=1}^{n}\left(A_{i j} * B_{i j}\right)}{\sum_{i=1}^{m} \sum_{j=1}^{n}\left(A_{i j}\right)^{2}}
$$

\subsubsection{Maximum Difference}

$$
M D=\max \left(\left|A_{i j}-B_{i j}\right|\right), i=1,2, \ldots m ; j=1,2, \ldots n
$$

\subsubsection{Normalized Absolute Error}

$$
N A E=\frac{\sum_{i=1}^{m} \sum_{j=1}^{n}\left(\left|A_{i j}-B_{i j}\right|\right)}{\sum_{i=1}^{m} \sum_{j=1}^{n}\left(A_{i j}\right)}
$$

\section{EXPERIMENTS AND DISCUSSION}

In this section we present our experiments and our inferences based on the results we obtained. Once the sample set of input image pairs were fused, the quality of the same were assessed for all fusion algorithms, discussed in section 2 with the image quality metrics, discussed in section 3 .

\subsection{Fused Images}

The experiments were performed based on 10 image sets. Each image set consisted of 11 images obtained by fusion using each of the algorithms. The images were, typically, multi focal and multi spectral images.

In this section we share one of the image sets we considered for our experiments (Fig. 1 through Fig. 7). Typically an image set would comprise of a pair of input images and the set of 11 fused images produced by each of the fusion algorithms. Here, we have a pair of input medical images, with one of them being a CT scan image and the other being MR scan image.

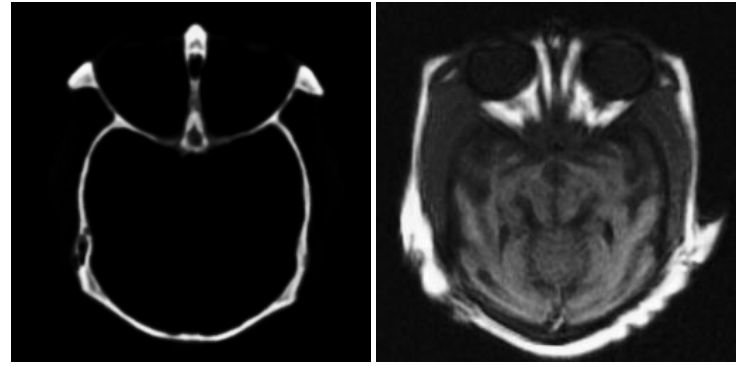

Fig 1: Pair of medical image input

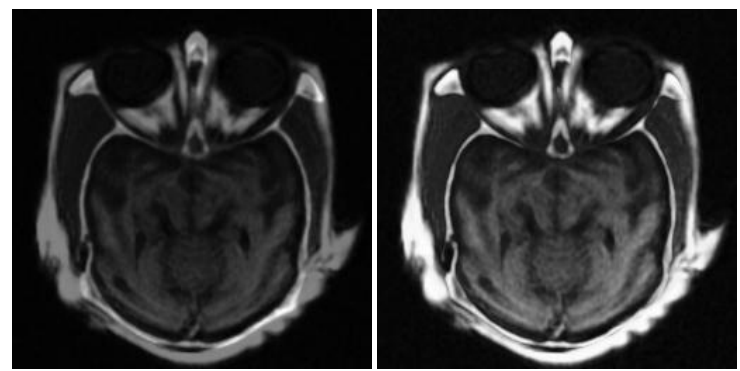

Fig 2: Fused Image by Average Method (left) and Maximum Method (right)

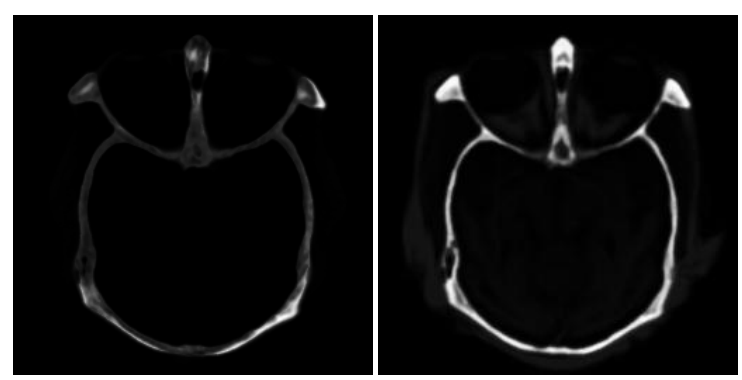

Fig 3: Fused Image by Select Minimum Method (left) and PCA method (right).

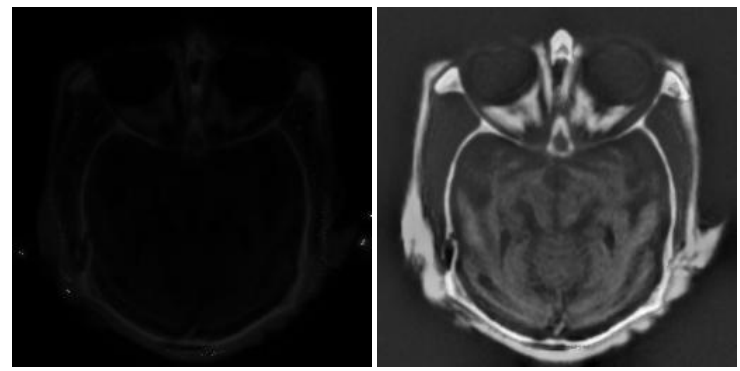

Fig 4: Fused Image by Ratio Pyramid Method (left) and Laplacian Pyramid method (right)

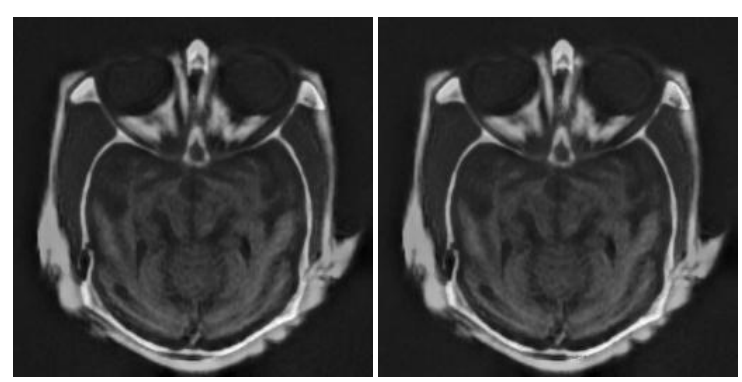

Fig 5: Fused Image by Select Gradient Pyramid Method (left) and FSD Pyramid Method (right) 


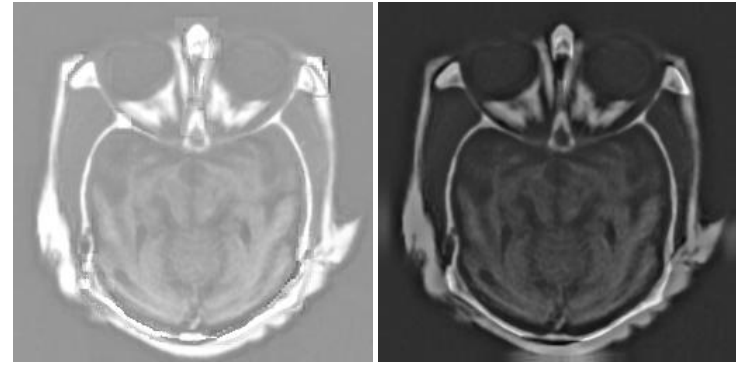

Fig 6: Fused Image by Select Morphological Pyramid Method (left) and DWT with Haar Method (right)

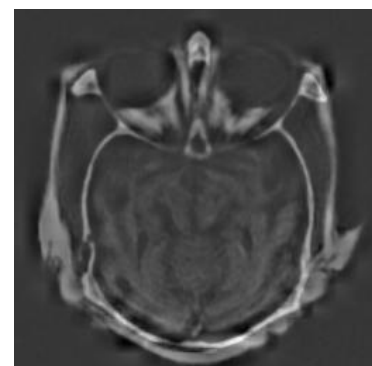

Fig 7: Fused Image by DBSS(2,2) Wavelet Method

Similarly, eleven other image sets were considered for our experiments. The image sets would, typically, be multi spectral images (as the above example) or multi focal images. The performance of the different fusion algorithms are evaluated using the metrics introduced in Section 3. However, these metrics are not trivially comparable and hence, the algorithms cannot be ranked. Such a multi-metric problem is the primary object of study for Pareto optimality. In the forthcoming subsection, we introduce the notion of Pareto optimality and present our algorithms to find the Pareto efficient algorithms.

\subsection{Pareto Optimality}

Pareto optimality is a commonly used tool in multi-objective optimization when the different objectives/metrics cannot be directly compared. In this subsection, without loss of generality, we consider objectives/metrics that need to be maximized. A good exposition of the Pareto optimality and the tools used to solve for Pareto optimality is found in [32]. Consider a set of image fusion algorithms, $\mathrm{A}=\left\{a_{1}, a_{2}, \ldots, a_{n}\right\}$. The vector valued function $f: \mathrm{A} \rightarrow \square^{\mathrm{m}}$ maps the space of algorithms to the space of objectives/metrics. functions $f_{1}, f_{2}, \ldots, f_{m}$. $f_{i}^{j}$ denotes the projection $\left[\mathrm{f}_{\mathrm{i}}, \mathrm{f}_{\mathrm{i}+1}, \mathrm{f}_{\mathrm{i}+2}, \ldots, \mathrm{f}_{\mathrm{j}}\right]$. We assume that each of the $m$ metrics is rounded to the nearest integer.

The two commonly used order for comparing vector quantities are the component-wise $(<)$ order and strict component-wise order $(<<)$.

$f\left(a_{m}\right)<f\left(a_{n}\right)$ iff $f_{i}\left(a_{m}\right) \leq f_{i}\left(a_{n}\right), \quad \forall i \quad$ and $f\left(a_{m}\right) \neq f\left(a_{n}\right)$. And $f\left(a_{m}\right) \ll<f\left(a_{n}\right)$ iff $f_{i}\left(a_{m}\right)<f_{i}\left(a_{n}\right), \forall i$.

An algorithm $\mathrm{a}^{\mathrm{p}} \in \mathrm{A}$ is Pareto efficient if there exists no other algorithm $z \in A$ such that

$f(z)<f\left(a^{p}\right)$. In a typical instance, there are many Pareto efficient algorithms. The set of Pareto efficient algorithms is called the Pareto frontier. We use Algorithm 1 to obtain this Pareto frontier. The vector function consists a sequence of scalar In Algorithm 1, the $\varepsilon$ 's are (m-1) dimensional vectors.
This algorithm can be efficiently implemented using matrix comparisons. We prove the Pareto Optimality of our algorithm. Algorithm 1 is a discrete version of the Haimes- $\varepsilon-$ relaxation method.

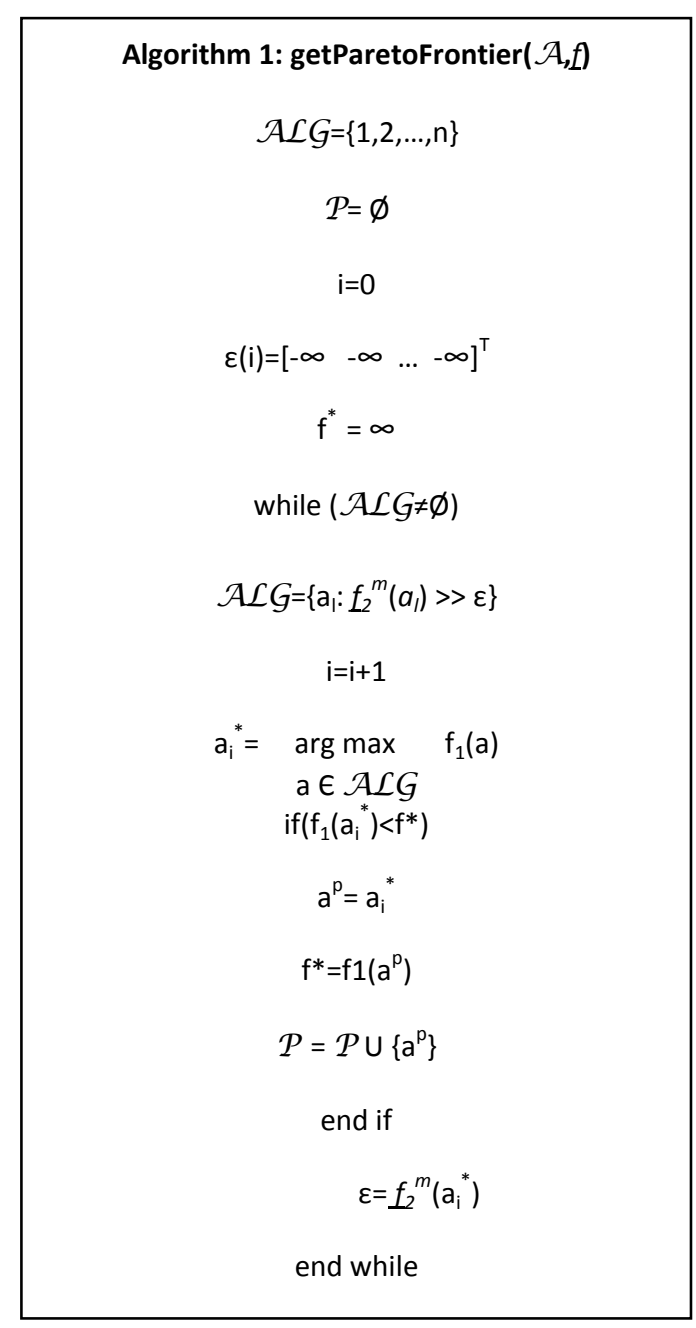

If $a_{j}{ }^{*}$ is a not weak Pareto solution, then there exists $z \in A$ and $\mathrm{z} \neq \mathrm{a}_{\mathrm{j}}{ }^{*}$ such that $f(\mathrm{z}) \gg>f\left(\mathrm{a}_{\mathrm{j}}{ }^{*}\right)$. Let $\varepsilon=\varepsilon^{\prime}$ at this iteration.

$$
\begin{aligned}
& f(\mathrm{z})>>f\left(\mathrm{a}_{\mathrm{j}}{ }^{*}\right)=>\mathrm{f}_{1}(\mathrm{z})>\mathrm{f}_{1}\left(\mathrm{a}_{\mathrm{j}}{ }^{*}\right) \\
& \mathrm{f}_{2}(\mathrm{z})>\mathrm{f}_{2}\left(\mathrm{a}_{\mathrm{j}}{ }^{*}\right)>\varepsilon^{\prime}(1) \quad \ldots \ldots(2) \\
& \cdot \\
& \cdot \\
& \mathrm{f}_{\mathrm{m}}(\mathrm{z})>\mathrm{f}_{\mathrm{m}}\left(\mathrm{a}_{\mathrm{j}}{ }^{*}\right)>\varepsilon^{\prime}(\mathrm{m}-1) \ldots \ldots(
\end{aligned}
$$

Equations $(2)-(m) \Rightarrow z$ is a feasible algorithm in ALG. Then Equation is a contradiction, since $a_{j}{ }^{*}$ is the maximizer for this feasible set of algorithms. Thus, $a_{j}{ }^{*}$ is weakly Pareto.

\section{Theorem 1:}

\section{$\left\{\mathrm{a}^{\mathrm{p}}\right\} \in \mathrm{P}$ is Pareto optimal}

From Lemma 1, $a^{\mathrm{p}}$ is a weak Pareto point. Let $\mathrm{a}_{1}{ }^{*}, \mathrm{a}_{2}{ }^{*}, \mathrm{a}_{3}{ }^{*}, \ldots$, $\mathrm{a}_{\mathrm{l}-1}{ }^{*}$ be the sequence of weak Pareto algorithms before the algorithm yields $a_{1}{ }^{*}=a^{p} . \Rightarrow f_{1}\left(a_{1}{ }^{*}\right)=f_{1}\left(a_{2}{ }^{*}\right)=\ldots=f_{1}\left(a_{1}{ }^{*}\right)=$ $\mathrm{f}_{1}\left(\mathrm{a}^{\mathrm{p}}\right)$. Then Let $\varepsilon=\varepsilon^{\prime}$ at this iteration.

Then, 


$$
\mathrm{a}^{\mathrm{p}}=\mathrm{a}_{1}{ }^{*}=\underset{\mathrm{a} \in \mathrm{A}}{\arg \max _{f_{2}^{\mathrm{m}}(\mathrm{a})} \mathrm{f}_{1}(\mathrm{a})},
$$$$
(\mathrm{m}+1)
$$

Suppose if $a^{p}$ is not Pareto efficient, then there exists $z \in A$ and $z \neq a_{j}{ }^{*}$ such that $f(z)>f\left(a^{p}\right)$. Since $a^{p}$ is the maximizer for Equation $(m+1)$, we have

$\mathrm{f}_{1}(\mathrm{z})=\mathrm{f}_{1}\left(\mathrm{a}^{\mathrm{p}}\right)$,

$f_{2}(z) \geq f_{2}\left(a^{p}\right)>\varepsilon^{\prime}(1)$,

$f_{m}(z) \geq f_{m}\left(a^{p}\right)>\varepsilon^{\prime}(m-1)$, where one of the inequality is true.

Let us assume, with out of loss of generality, $f_{2}(z)>f_{2}\left(a^{p}\right)$. Let $\varepsilon "=f_{2}^{m}\left(\mathrm{a}^{\mathrm{p}}\right)=\left[\mathrm{f}_{2}\left(\mathrm{a}^{\mathrm{p}}\right) \varepsilon " '(2) \varepsilon "(3) \ldots \varepsilon " '(\mathrm{~m}-1)\right]$, be the threshold for the next iteration.

For the next iteration, we obtain

$$
\begin{gathered}
\mathrm{a}_{1}{ }^{*}=\underset{\mathrm{a} \in \mathrm{A}}{\arg \max } \\
f_{2}^{\mathrm{m}}(\mathrm{a})>>\varepsilon^{\prime},
\end{gathered}
$$

Now, $\mathrm{a}_{1}{ }^{*}$ must be $\mathrm{z}$, because it satisfies the constraints. But this is a contradiction. Hence $\mathrm{a}^{\mathrm{p}}$ is indeed Pareto efficient. ....QED

Thus, Algorithm 1 returns the Pareto frontier. All other algorithms that are not a part of the Pareto frontier are dominated by a better algorithm in the Pareto frontier. In other words, our Pareto method sieves out those algorithms that are inefficient in the Pareto sense.

The image metrics readings for the twelve image sets were averaged and the Pareto Frontier was calculated on this averaged matrix. The method projected DWT with Haar based fusion method to be efficient, compared to the others, based on the reading produced by the image quality metrics. The method also revealed Morphological Pyramid method to be a dominated algorithm (inferior algorithm).

\subsection{Assessment based on Visual Perception}

We also attempted to verify and confirm the results produced based on the image quality metrics by assessing the fused image based on its visual appearance. Three image sets were selected for the assessment. Ten respondents were selected in random and were asked to select the image they visually understood to be of the best quality of the 11 fused images in each of the three sets. Table 1 exhibits the assessment made by the respondents; the algorithm corresponding to the image they found best in quality in each of the three image sets is listed in the table. It can be noted that of the 30 assessments made, 19 times $(63.33 \%)$ the fused image by DWT with Haar based fusion method was adjudged the best (Fig 8). It can also be inferred from the table that each respondent definitely adjudged Haar based fused image best in either of the image sets.

It can be noted that though Morphological pyramid method is quite an advanced method in comparison to some of the other algorithms considered, it was assessed inferior to them. It was understood that through the process of image opening and image closing involved in the morphological pyramid based fusion, the sharpness of the edges of the objects in the images were enhanced, but at the cost of the overall quality of the image. A foggy effect was produced on the overall image which gave in the visual feel of the image being rather painted. Figure 9 exhibits the foggy effect in the morphological pyramid based fused image, comparing it with DWT with Haar based fused image, which was rated the best. In Ratio Pyramid based fusion, which was rated inferior after Morphological pyramid method, during the pyramid formation at each level of fusion, the difference matrix was arrived upon based on the ratio between the corresponding pixels of the input images and the filtered image. Considering the ratio of the pixel intensity does not physically contribute to an explanation of enhancement of the sharpness of an image.

Table 1: Assessment made by the respondents

\begin{tabular}{|c|c|c|c|}
\hline \multirow{2}{*}{ Respondent } & \multicolumn{3}{|c|}{ Image Set } \\
\hline A & Grad Pyd & Haar & Haar \\
\hline B & Haar & Haar & Haar \\
\hline C & Grad Pyd & DBSS & Haar \\
\hline D & Haar & Haar & DBSS \\
\hline E & Haar & FSD Pyd & Haar \\
\hline F & Haar & Haar & Haar \\
\hline G & Haar & Haar & Haar \\
\hline H & Grad Pyd & Haar & DBSS \\
\hline I & Haar & FSD & DBSS \\
\hline J & Haar & Haar & DBSS \\
\hline
\end{tabular}

Table 2 depicts the sample reading produced by the 9 image quality metrics for each of the 11 algorithms. Similarly, the readings produced for all the ten image sets were taken into consideration.

\section{SOME IMPLEMENTATION ISSUES}

The eleven image fusion algorithms were implemented using Visual C++ 6.0 developing a toolkit called ImFus. Fig 10 depicts the graphical user interface developed for the toolkit.

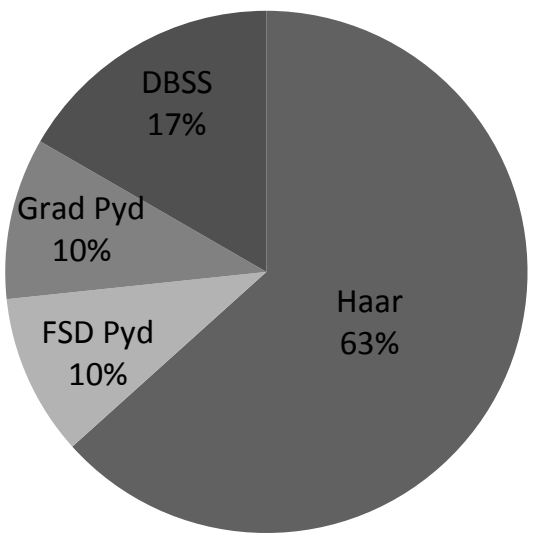

Fig 8: Distribution of algorithms adjudged best based on visual quality. 


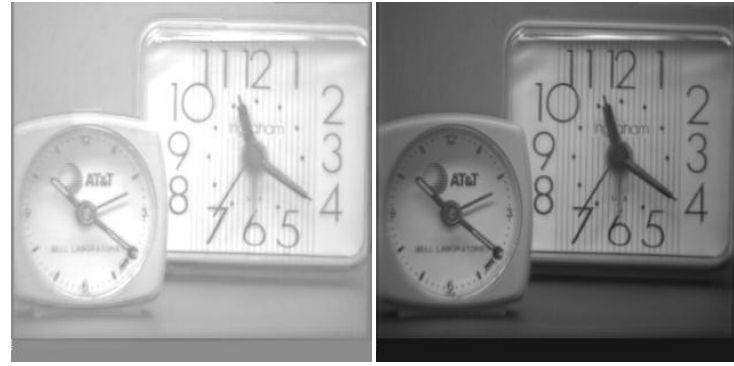

Fig 9: Foggy effect in Morphological Pyramid based fused image (left) in comparison with DWT Haar based fused image.

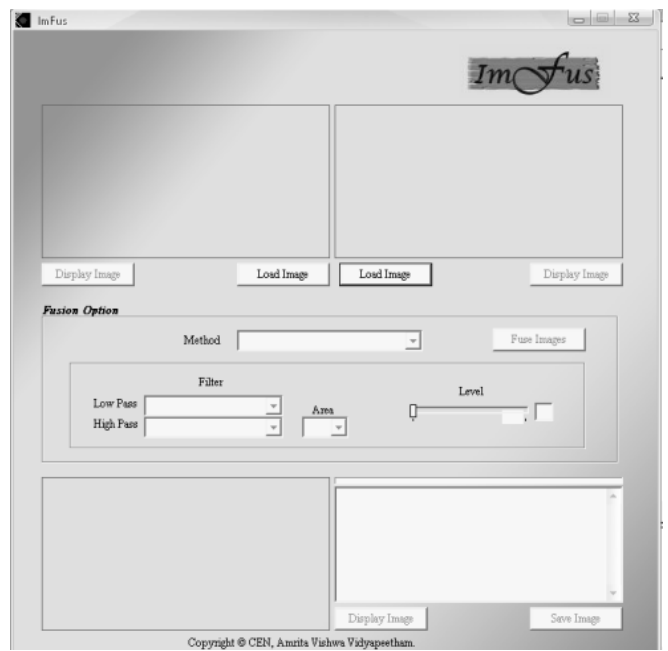

Fig 10: GUI for ImFus Toolkit

There were some implementation issues to be addressed for the development of the toolkit using $\mathrm{C}++$. Some of the major issues are discussed in this section.

The eleven fusion algorithms considered here are pixel based fusion methods. So there was a need to access each input image as an image matrix with each element being the pixel intensity value, thus making it possible to manipulate the pixel intensities of the images. CImg [8] - a C++ Template Image library was used for the purpose.

Though CImg provided a vast set of image processing functions, it was mainly utilized only for

- $\quad$ Reading an image from a file as a matrix

- Accessing each pixel value from the image matrix

- Displaying the intermediate images during the process of fusion

- Displaying the images in their actual sized in a new window.

- Permanently saving the image
The toolkit supported two uncompressed image formats, namely, .bmp and .raw format. A bmp header was added to the raw images and saved only as a .bmp so as to make it convenient for the user to open and view the image even without the help of the toolkit. Otherwise, to open a raw image, information at least like the image dimensions and the bit size are to be provided.

In case of the Pyramid based and DWT Transform based fusion, the extent of the level of fusion was decided upon based on the dimensions of the input images. Though the user is provided with the option to select the level of fusion to be performed, the option provided to him was based on the pixel rows and columns in the input pair of images. The level restriction was imposed such that the decimation process does not cut the image down below the size of 10 , either row wise or column wise. It was noticed, based on numerous trials, that when the image matrix dimension went below this size of 10 , further filtering and decimation did not make much of a difference in the quality of the final fused image.

\section{FUTURE SCOPE}

The work performed does hold scope for further advancements as a lot of research is happening in the field. The following are some proposed practical advancements possible in the project:

- Multi Wavelets [12] [28] based image fusion can be performed to achieve a better image fusion quality. [12] explains the efficiency of multi wavelets over the usual DWT methods in fusing images involved in remote sensing. The same can be applied in this project too and can be verified based on the image quality metrics developed.

- The image fusion quality has been assessed based on optical image sets with respect to a a perfect image. The efficiency of the fusion can be better assessed if the same could be performed on many more.

multivariate images. The same could not be done due to lack of such set of test sample multivariate images

- A learning algorithm like neural networks and more specifically Support Vector Machine [13] could be devised in assigning weightage to the image quality metrics so as to assess them. A more extensive number of image sets could be considered initiating a learning process using SVM, based on which the metrics could be provided with weighted ranks. This would again require more set of sample images.

- Image Registration [23] has not been incorporated in the work. Image Registration / Image Alignment will certainly enhance the efficiency of the project as vast set of even unregistered images can be considered as set of input images. It would also help in possibility of more set of sample test/perfect images made available for assessing the image fusion algorithms.

- The ImFus Toolkit now looks into considering only two input images to be fused. An option to load and fuse more than two images at the same time can also be easily incorporated into the project. An option could be provided to the user on to select the number of input images available 
Table 2: Image metrics readings for one image set.

\begin{tabular}{|c|c|c|c|c|c|c|c|c|c|}
\hline & MSE & PSNR & AD & SC & NCC & MD & NAE & LMSE & SSIM \\
\hline Average & 177.28 & 25.64 & -9.57 & 0.85 & 1.08 & 82 & 0.09 & 0.2 & 95.29 \\
\hline Maximum & 116.55 & 27.47 & -1.22 & 0.98 & 1.01 & 22 & 0.04 & 0.65 & 91.22 \\
\hline Minimum & 304.96 & 23.29 & -13.78 & 0.81 & 1.11 & 175 & 0.13 & 0.53 & 93.16 \\
\hline PCA & 177.17 & 25.64 & -9.51 & 0.85 & 1.08 & 84 & 0.09 & 0.2 & 95.24 \\
\hline FSD Pyd & 157.12 & 26.17 & -10.4 & 0.09 & 1.08 & 96 & 0.09 & 0.13 & 97.05 \\
\hline Lap Pyd & 305.43 & 23.28 & -16.47 & 0.08 & 1.1 & 92 & 0.13 & 0.11 & 96.15 \\
\hline Grad Pyd & 146.26 & 26.48 & -9.57 & 0.86 & 1.07 & 74 & 0.08 & 0.11 & 97.36 \\
\hline Rat Pyd & 166.11 & 25.93 & -0.58 & 1 & 1 & 56 & 0.06 & 0.48 & 90.06 \\
\hline Mor Pyd & 1855.1 & 15.45 & -39.29 & 0.64 & 1.23 & 104 & 0.32 & 0.45 & 86.52 \\
\hline Haar & 244.79 & 24.24 & -14.39 & 0.83 & 1.09 & 84 & 0.12 & 0.1 & 96.06 \\
\hline DBSS & 359.2 & 22.58 & -17.07 & 0.82 & 1.1 & 66 & 0.14 & 0.09 & 95.09 \\
\hline
\end{tabular}

\section{CONCLUSION}

The eleven image fusion techniques were implemented using Microsoft Visual C++ 6.0. The fusion was performed on twelve sets of input pair of images. The fused images were verified for their quality based on a perfect image in each of the sets. A set of nine image metrics were implemented to assess the fused image quality. The fused images of each set were also assessed based on their visual quality by ten respondents selected in random. The quality assessment based on the image metrics developed and visual perception was compared to assess the credibility of the image metrics.

In the total of eleven image fusion techniques, three very basic fusion techniques were Averaging Method, Maximum Selection Method and Minimum Selection Method, five pyramidal methods were FSD Pyramid, Laplacian Pyramid, Gradient Pyramid, Ratio Pyramid and Morphological Pyramid Methods and two of basic wavelet methods were Haar Wavelet and DBSS $(2,2)$ Wavelet Methods. The readings produced by the 9 image metrics developed - MSE, PSNR, SC, NCC, AD, MD, NAE, LMSE and SSIM, were used to assess the best fusion algorithm (in terms of the quality of the fused images) using Pareto optimality method. DWT with Haar based fusion method was assessed best. The assessment saw that the fused images produced by Morphological Pyramid Method were the rated most inferior in quality.

The algorithms were also assessed based on the visual quality of the fused images. Ten people were selected, in random, to

visually assess the fused images produced in each of the 3 sets and were asked to pick out the best and worst image they found in each image set. The results here validated the results produced based on image metric readings. DWT with Haar was rated $63.33 \%$ times, much higher that the rating given to the other algorithms. Similarly the results also matched as Morphological pyramid rated inferior in visual quality.

\section{ACKNOWLEDMENTS}

We sincerely thank Kiran Kumar Somasundaram, ISR, University of Maryland, College Park, USA, for his significant contribution in helping us to assess the fusion algorithms using Pareto optimality. We also express our gratitude to Mr. Ajay Vadakkepatt for his constant support throughout our study and assessment.

\section{REFERENCE}

[1] J Zeng, A Sayedelahk, T Gilmore, P Frazier, M Chouika, "Review of Image Fusion Algorithms for Unconstrained Outdoor Scenes", Proceedings of the 8th International Conference on Signal Processing, Volume 2, pages 1620, 2006.

[2] Farhad Samadzadegan, "Fusion Techniques in Remote Sensing”. http://www.igf.uniosnabrueck.de/mitarbeiter/ schiewe/papers/43.pdf

[3] E H Adelson, C H Anderson, J R Bergen, P J Burt, J M Ogden, "Pyramid Methods in Image Processing"

[4] Florence Laporterie, Guy Flouzat, "Morphological Pyramid Concept as a Tool for Multi Resolution Data Fusion in Remote Sensing", Integrated Computer-Aided Engineering, pages 63-79, 2003

[5] Peter J Burt, Edward Adelson, "Laplacian Pyramid as a Compact Image Code", IEEE Transactions on Communications, Vol Com-31, No. 4, April 1983.

[6] FuseTool - An Image Fusion Toolbox for Matlab 5.x, http://www.metapix.de/toolbox.htm

[7] Matifus - A Matlab Toolbox for Image Fusion, http://ftp.cwi.nl/pauldz/Codes/MATIFUS

[8] CImg - C++ Template Image Processing Library. http://cimg.sourceforge.net/

[9] The Online Resource for Research in Image Fusion www.imagefusion.org

[10] Z. Wang, A. C. Bovik, H. R. Sheikh, and E. P. Simoncelli, "Image quality assessment: From error visibility to structural similarity“, IEEE Transactions on Image Processing, vol. 13, no. 4, pp.600-612, Apr. 2004

[11] Yang C., Zhang J., Wang X., Liu X, "A novel similarity based quality metric for image fusion". Information Fusion 9(2): 156-160, 2008. 122 
[12] Tan Na, Ehlers Manfred, Wanhai Yang, "Remote Sensing Image Fusion with multiwavelet Transform", SPIE proceedings series 2004, Vol. 5574, pp. 14-22. ISBN 0- 8194-5521-0

[13] "Soman KP, R. Loganathan, Vijaya MS, Ajay V and Shivsubramani K. Fast Single-Shot Multiclass Support Vector Machines and Perceptrons", In Proc. of the International Conference on Computing: Theory and Applications (ICCTA'07), pages 294-298. IEEE Computer Society Press, March 2007

[14] Y. Wang, Z. You, J. Wang, SAR and optical images fusion algorithm based on wavelet transform, in proceedings of the 7th International Conference on Information Fusion (Fusion 2004), Stockholm, Sweden, June 28 - July 1, 2004, International Society of Information Fusion (ISIF), 644-648

[15] C. Xydeas and V. Petrovic, Objective pixel-level image fusion performance measure, Proceedings of SPIE, Vol. 4051, April 2000, 89-99

[16] J. J. Lewis, R. J. O'Callaghan, S. G. Nikolov, D. R. Bull, C. N. Canagarajah, Regionbased image fusion using complex wavelets, Proceedings of the 7th International Conference on Information Fusion (Fusion 2004), Stockholm, Sweden, June 28 - July 1, 2004, International Society of Information Fusion (ISIF), 555-562

[17] F. Sadjadi, "Comparative Image Fusion Analysais", IEEE Computer Society Conference on Computer Vision and Pattern Recognition, Volume 3, Issue , 20-26 June 2005 Page(s): $8-8$

[18] Y. Chen and R. S. Blum, "A new automated quality assessment algorithm for night vision image fusion," in Proceedings of the Conference on Information Sciences and Systems (CISS), Baltimore, MD, March 2007.

[19] R. S. Blum, "The Cramer-Rao bound applied to image fusion," in Proceeding of the $8^{\text {th }}$ International Conference on Information Fusion, Philadelphia, PA, July 2005.

[20] Yin Chen and R. S. Blum, "Experimental tests of image fusion for night vision," in Proceeding of the 8th International Conference on Information Fusion, Philadelphia, PA, July 2005.
[21] Z. Xue and R. S. Blum, "Concealed Weapon Detection Using Color Image Fusion," the 6th International Conference on Image Fusion, Queensland, Australi, July 8-11, 2003.

[22] Z. Xue, R. S. Blum and Y. Li, "Fusion of visual and IR images for concealed weapon detection," invited paper at International Conference on Information Fusion, Annapolis, Maryland, July 2002. 123

[23] Z. Zhang and R. S. Blum, "Image registration for Multifocus image fusion", SPIE AeroSense, Conference on Battlefield Digitization and Network Centric Warfare (4396-39), Orlando, FL, April 2001.

[24] Zhang Zhong, "Investigations on Image Fusion,", PhD Thesis, University of Lehigh, USA. May 1999

[25] Zhiyun Xue, Image Fusion, PhD Thesis, University of Lehigh, USA. August 2006.

[26] Jinzhong Yang, Image Fusion, PhD Thesis, University of Lehigh, USA. August 2006.

[27] Z. Zhang and R. S. Blum, "Image Fusion for a Digital Camera Application" Invited paper at Asilomar Conference on Signals, Systems, and Computers, pp. 603-607, Monterey, CA, 1998.

[28] Chui C K, Jian-ao L, "A Study of orthonormal multi wavelets", Applied Numerical Mathematics, Vol. 20, Number 3, March 1996, pp. 273-298(26)

[29] Lindsay I Smith, "A Tutorial on Principal Component Analysis",http://www.cs.otago.ac.nz/cosc453/student_tut orials/principal_components.pdf

[30] "Principal Component Analysis", http://www.cse.unr.edu/ bebis/MathMethods/PCA/lectur e.pdf

[31] Luc Vincent, "Morphological Area Openings and Closings for Greyscale Images Proc. NATO Shape in Picture Workshop, Driebergen, Springer-Verlag, pp. 197208, September 1992.

[32] Ehrgott M, “Multicriteria Optimization”, Springer, 2000. 